\title{
How do general practitioners conceptualise self-harm in their older patients?
}

\author{
A qualitative study
}

Anne PF Wand, Carmelle Peisah, Brian Draper, Henry Brodaty

\section{Background and objectives}

Little is known about how general practitioners (GPs) conceptualise self-harm in older people. The aim of this study was to explore GPs' understanding of the reasons for recent self-harm in an older patient.

\section{Method}

Short questionnaires were sent to the GPs of patients aged 80 years or older who had recently self-harmed and were participants in a qualitative study about self-harm. Questions evaluated GPs' understanding of the self-harm. Thematic analysis was used to identify and analyse themes.

\section{Results}

Thirteen GP responses were analysed. GPs could identify multiple factors contributing to self-harm in their patients but did not see a role for themselves in addressing these issues. They feared repetition of self-harm if these underlying contributory factors, including depression, did not change.

\section{Discussion}

Targeted education and practical options for GPs regarding management of the issues underlying self-harm in older people are needed. Families and carers may be underused allies in management. These strategies may serve to counter therapeutic nihilism and clinician isolation.
GENERAL PRACTITIONERS (GPS) are at the front-line of healthcare for older people. Most older people who have died by suicide have seen a GP in the preceding three months, ${ }^{1}$ representing a potential opportunity to intervene, an imperative in an ageing population with high rates of suicide, particularly among men. ${ }^{2}$

Self-harm and suicide are closely related in older people, with common risk factors such as depression, pain and social isolation. After an episode of selfharm, there is a high risk of subsequent suicide. Self-harm, which is any selfinjurious act carried out by a person regardless of intention, may be direct (eg cutting, overdose) or indirect (eg refusing to eat or drink). ${ }^{3}$ A recent psychological autopsy study (which uses informants to reconstruct the lived experience and circumstances of the deceased leading up to their suicide) demonstrated that GPs and carers know much about risk indicators for suicide in their patients and loved ones, with carer knowledge being greater but, often, not communicated to GPs. ${ }^{4}$ Several of these risk factors for suicide are potentially remediable and GPs are well placed to identify and manage them.

The understanding and attitudes of GPs to self-harm in older patients have been little studied. One study compared physician responses to hypothetical vignettes involving an employed man aged 38 years and a retired man aged 78 years, both of whom were depressed and suicidal, to explore age biases in management. ${ }^{5}$ Physicians detected depression and suicide risk in both vignettes, but they were less willing to treat the older patient and more likely to evaluate suicidal ideation in the older patient as more normal, acceptable and rational. ${ }^{5}$
A qualitative study found that primary care practitioners (GPs and nurses) considered depression in older people as part of a range of psychosocial issues, such as loneliness, dependence and poor social support, rather than a distinct diagnostic category, and that depression was 'justifiable' and 'understandable'. ${ }^{6}$ Associated with this was the sense that 'nothing could be done' (therapeutic nihilism), lack of active management, and limited expectations of treatment. ${ }^{6}$ While the primary care practitioners identified depression as being within their scope of practice, they reported that they lacked skills, resources and referral pathways for these patients. Others postulated that reasons for the under-recognition and under-treatment of depression in old age ${ }^{7}$ include negative attitudes of GPs, inadequate training and ineffective treatment approaches. ${ }^{8}$

Our primary aims were to explore qualitatively GPs' understanding of factors contributing to self-harm in their older patients who had recently self-harmed and how these factors could be addressed; and perceptions of the intent of the selfharm and their concerns about repetition. Secondary aims were to identify potential targets for GP education.

\section{Methods}

Ethics approval was granted by South Eastern Sydney Local Health District Human Research Ethics Committee and University of New South Wales (application number 16/269).

Patient participants were aged 80 years and over, had self-harmed in the previous month, and had been recruited to a qualitative study of self-harm in which 


\section{Table 1. The clinical and demographic characteristics of patients who had} self-harmed

\begin{tabular}{|c|c|}
\hline Characteristic & $\mathrm{n}=25$ \\
\hline Age & Mean 86 , range (80-93) \\
\hline Gender & 12 (48\%) female \\
\hline Non-English speaker & $11(44 \%)$ \\
\hline \multirow{2}{*}{$\begin{array}{l}\text { Living arrangement prior to } \\
\text { self-harm }\end{array}$} & Home 18 (72\%) \\
\hline & Residential aged care facility 7 (28\%) \\
\hline \multirow{2}{*}{$\begin{array}{l}\text { Involved with mental health } \\
\text { service/clinician immediately } \\
\text { prior to the self-harm }\end{array}$} & $6(24 \%)$ \\
\hline & $3(12 \%)$ had recently refused mental health care \\
\hline \multirow[t]{7}{*}{ Type of self-harm } & Overdose 12 (48\%) \\
\hline & Cutting $5(20 \%)^{*}$ \\
\hline & Biting $1(4 \%)$ \\
\hline & Refusal to eat $3(12 \%)$ \\
\hline & Suffocation $1(4 \%)$ \\
\hline & Hitting oneself $3(12 \%)^{*}$ \\
\hline & Carbon monoxide poisoning 1 (4\%) \\
\hline \multirow{4}{*}{$\begin{array}{l}\text { Hospital admission post self- } \\
\text { harm (where discharged from) }\end{array}$} & Total: $22(88 \%)$ \\
\hline & Psychiatry 13 (52\%) \\
\hline & Geriatrics 6 (24\%) \\
\hline & General medicine $3(12 \%)$ \\
\hline Previous self-harm & $11(44 \%)$ \\
\hline \multirow[t]{7}{*}{ DSM-5 diagnosis } & Major depression 10 (40\%) \\
\hline & Alcohol use disorder 2 (8\%) \\
\hline & Minor depression 1 (4\%) \\
\hline & Delirium 1 (4\%) \\
\hline & Anxiety disorder $1(4 \%)$ \\
\hline & Very late onset schizophrenia like psychosis $1(4 \%)$ \\
\hline & No psych illness or cog imp 1 (4\%) \\
\hline Dementia & $10(40 \%)$ \\
\hline Mild cognitive impairment & $9(36 \%)$ \\
\hline
\end{tabular}

they were interviewed by a psychiatrist (AW) about their reasons for self-harm. Patients nominated their primary GP and consented to them being contacted. This study reports data obtained from the GP study. All GPs were contacted by email or facsimile. A questionnaire was developed and included basic demographic information (age, gender, years they have known the patient, how they learned about the self-harm) and open-response questions eliciting their perception of contributing factors to the patient's self-harm, how these factors could be addressed and by whom, their reflections on the intent or purpose of the self-harm and thoughts about the possibility of repetition. Consent to participate was implied by completion of the questionnaire. If not returned, the questionnaire was re-sent. GPs who did not respond were contacted to confirm receipt of the questionnaire and offered telephone completion of the questionnaire. All responders chose written responses.

\section{Data collection and analysis}

The study design was qualitative. Thematic analysis was used to identify, analyse and describe patterns in GP responses to open questions. ${ }^{9}$ For each of the open-response questions the text was analysed line by line and then grouped into themes and subthemes. An iterative process was used to re-examine previously analysed data in light of emergent themes. Two researchers (AW and $\mathrm{CP}$ ) independently analysed the GP responses. Where there was disagreement, data were re-examined and discussed until consensus was reached. Descriptive statistics were used for the demographic characteristics of the GPs and patients.

\section{Results}

Twenty-five patients who had selfharmed within the previous month consented to take part in the study. Clinical and demographic characteristics are shown in Table 1 . Thirteen $(13 / 25$, $52 \%)$ GPs returned questionnaires. Reasons for declining to participate included lack of interest, not having seen the patient for a while, feeling uncomfortable about commenting, concerns about the validity of patients' consent for non-English speaking patients, citing 'sensitive issues', lack of time and immediate clinical demands.

The mean age of the GPs was 51 years (range 43-63). Nine (69\%) were male and most had known their patient for several years (range two weeks to $>30$ years, mean 15 years excluding the GP who had assumed care of his patient two weeks previously). Two of the 13 GPs did not know that their patient had self-harmed; the remaining 11 had been informed by hospital staff, either directly (8) or via discharge summaries (3); one GP was additionally informed by a patient's family member.

\section{Qualitative analysis}

The following themes emerged from the analysis of the responses: 


\section{Factors contributing to self-harm}

\subsection{Physical and mental illness}

Only two of the 13 GPs failed to identify reasons for self-harm. Typically, GPs identified a raft of possible reasons, including both mental and physical illnesses such as depression, anxiety, delirium and Alzheimer's dementia, sometimes several in one individual. One GP listed 'advanced age' as a contributing factor among a mix of other factors, including chronic pain, depression and early cognitive impairment. A typical list was:

Chronic pain; coronary disease; metastatic cancer; fungating cancer-very unsightly, painful, bleeding smelly; worsening arthritis/immobility ... - GP 21

GPs were less likely to identify cognitive and mood disorders than the psychiatric interviews.

\subsection{Psychosocial problems}

Social issues, including stress as a carer, isolation, loneliness and bereavement, were also identified. Threats to independence were mentioned as precipitants to self-harm and seen as potentially ameliorable, and moving to residential care was seen as both a trigger to, and a consequence of, self-harm:

\section{Concerned about moving to a nursing home ... Addressed by reassurance and organising a carer-NOT moving to a $\mathrm{NH}$ [nursing home] - GP 7}

Patient was sent [to a nursing] home for future care. - GP 20

\subsection{Hopelessness}

Loss of hope, especially that engendered by other practitioners, was identified as a trigger for self- harm. GPs identified the importance of maintaining hope for their patient and how this could be achieved:

Has deterioration of vision. Was told via ophthalmologist that week that vision will deteriorate more to total blindness. - GP 5

A tentative suggestion of how to address the hopelessness engendered by others was offered:
Stating [that it was] hard to assess degree of blindness in future, rather than say will be totally blind? - GP 5

Conversely, some GPs shared, and possibly fuelled, their patient's sense of hopelessness:

Nothing could be done - he refused cancer treatment, counselling, antidepressants ... It is unlikely that antidepressants would have helped him anyway. - GP 21

\section{How to address the contributing factors to self-harm}

\subsection{Someone else}

An emergent theme regarding who could address contributing factors was 'someone else' (or 'not me'). GPs generally did not identify themselves as having a role in coordinating their patient's care in relation to self-harm. Only two GPs thought they might have a role in discussing mental health issues with the patient. Another suggested his patient would have received more consistent and continuous care had they seen the same GP at each visit. GPs listed specialists (eg ophthalmologists, psychiatrists), the family, hospital pain clinics, community care providers, dementia support services and local mental health services as potentially helpful to intervene. One GP could not identify anyone who could help the patient.

\subsection{Helplessness: I don't know what to do next}

Another emergent theme was a perceived lack of treatment strategies. Few specific treatments, such as reassurance and education, were identified, but it was unclear whom GPs thought would be the providers. Practical suggestions were made, including pharmacy-packaged medications and increasing supervision. There were few references to any psychological strategies. One GP asked:

\section{How do you deal with depression? - GP 19}

Not all GPs felt powerless. Some had a clear formulation of the problem and how risk of repetition could be mitigated:
Early identification of delirium and treat any reversible causes. Early attention to any patient call for help. Provide psychological intervention if patient still has insight or receptive. - GP 1

Another GP suggested:

\section{Coping skills to deal with medical issues} - GP 17

\section{Intent of the self-harm}

The GPs' perceived purpose or intent of the patient's self-harm included the wish to die, a cry for help, an impulsive behaviour, or a direct expression of depression or frustration. A number of GPs indicated they did not know what the intent of the self-harm might have been.

\section{Repetition of self-harm}

\subsection{If I can't fix the contributing factors} they will self-harm again

Most GPs (8/13) were concerned that their patient would self-harm again, particularly if supportive strategies were withdrawn, or the original triggers and contributory factors persisted, especially depression and isolation. Difficulties identifying and treating depression were particularly prominent:

[Referring to a patient with dementia and recurrent delirium] Hard to identify depression-GP 1

Ongoing rather resistant depression, social isolation-GP 11

She remains depressed, has low motivation to change and still lives alone ... - GP 2

In one case the self-harm had continued:

\section{Patient is still refusing eating - GP 19}

GPs who were not concerned about repetition felt that the contributing factors to the self-harm had been definitively addressed, cited the absence of any previous self-harm, or noted the patient had promised not to self-harm again.

Impulsive action. Spoke to her again. Will not do it. - GP 17 


\section{Discussion}

To the best of our knowledge, this is the only qualitative study of GPs of older patients who have self-harmed and survived. Most GPs, having known their patient for well over a decade, recognised the often multiple psychological, social and medical factors underlying self-harm, although their role in addressing these factors was less clear.

Key themes that emerged were GPs' sense of hopelessness and the perception that they had neither a leading role coordinating nor providing care for older patients with self-harm and often complex medical and social needs. Some GPs, seemingly isolated and overwhelmed by their patient's difficulties, expressed therapeutic nihilism, a known impediment to managing depression and suicide in later life. ${ }^{6,10}$ Such GP responses may have reflected feelings of guilt or grief at being unable to prevent their patient's selfharm. Consistent with previous research, while GPs identified numerous potential contributing factors to the self-harm, most nominated other clinicians, services or family to address the issues. ${ }^{11}$ This may have been feasible if there were a close and continuing relationship between GP, family and/or mental health services; our data suggest otherwise. Only one family member communicated the self-harm to the GP; most (19/25, 76\%) of the older patients in this study were not being seen by mental health services prior to their self-harm.

We do not know whether GPs contacted families. It is possible that this did not occur because of misunderstandings about the limits of confidentiality in the therapeutic relationship, although selfharm and suicide risk are valid reasons for waiving confidentiality. Next-of-kin know much about their relatives' suicide risk indicators, but rarely communicate this information to GPs. ${ }^{4}$ This represents a missed opportunity to identify patients who may have disclosed suicidal ideation to carers but not health professionals, ${ }^{12,13}$ or may have other potentially remediable suicide risk factors. ${ }^{4}$ Communication with carers could also have provided support, instilled hope and validated the family's role. ${ }^{4,14}$ Similarly, few GPs mentioned referral to, or communication with, mental health services, which could have been helpful especially for patients with apparent treatment resistance.

We confirmed that, often, when depression is identified in older people, GPs lack confidence and feel a sense of therapeutic helplessness. ${ }^{6,7,15}$ GPs highlighted difficulty identifying depression in people with cognitive impairment, and lack of knowledge and skills in treating depression in older people previously identified..$^{5,8,10}$

Dementia and cognitive impairment were similarly under-detected by GPs or perhaps not recognised as relevant to self-harm, which is in contrast to the psychiatrist's assessment. What impact the presence of cognitive impairment/ dementia has on GP concepts of self-harm is unknown.

One way to address these barriers would be through better education on cognitive impairment and depression in later life, particularly non-pharmacological options. The recognition of patients' hopelessness as an important risk factor should also be highlighted. The lack of GP references to psychological strategies to improve coping or treat mental illness was surprising, especially given the Australian Government's subsidised scheme for improving access to psychological treatments - the Better Access to Mental Health Care Program. ${ }^{16}$ However, patients in residential aged care facilities $(28 \%$ in this study) cannot access psychologists through the Medicare Benefits Scheme, but people with dementia and self-harm may access behavioural assessment and management programs through Dementia Support Australia. ${ }^{17}$ Another explanation for the infrequent mention of psychological strategies may have been the high proportion of non-English speaking patients (44\%), a recognised barrier to accessing psychological interventions.

The GP responses indicate the value of long-term therapeutic relationships with older patients. Their responses suggested warmth, empathy and compassion for the challenges facing their patients who had self-harmed, although possibly to the point of over-identification with their hopelessness. This holistic understanding and therapeutic alliance confirms what patients want from primary care in relation to depression. ${ }^{18}$ An Australian study also found that the empathy and openness of GPs to discussing emotional concerns in their older patients played a pivotal part in reducing ideas of selfharm, perhaps more so than treatment of depression. ${ }^{19}$ Patients have emphasised the importance of interpersonal aspects of their interactions with GPs, including empathising, listening and understanding, along with technical competence. ${ }^{18}$

\section{Reflexivity, strengths and limitations}

There were a number of limitations to this exploratory pilot study. First, the brief questionnaire format by correspondence (a more superficial qualitative typology), together with the small numbers, precluded a more comprehensive exploration of GP perspectives and data saturation. Second, in such a sensitive area, there may have been responder bias - it is possible that those with more interest in, and understanding of, selfharm replied, thereby leading us to overestimate GP competency. Social bias in responses is possible, ${ }^{20}$ although inaccurate reconstruction of events and attributions are less likely in this study, with data gathering so proximate to the self-harm.

Two of the authors were involved in the care of some of the patients recruited to the study. Therefore, they may have had contact with the participants' GPs, potentially influencing their responses. Another limitation is that GP responses might also have been enriched by interactions with mental health services following the self-harm. However, the converse may be true, that involvement with secondary health services impeded GPs' connection with their patients and their families - for example through blame, poor communication, or lack of collaboration.

\section{Implications for general practice}

Potential targets for GP education include:

- management of depression in older people, particularly when comorbid 
with cognitive disorders and suicidal ideation ${ }^{5,6,8,21}$

- instilling hope and optimism in GPs regarding treatment of depression and self-harm in older people, while at the same time exploring GPs' self-blame and the realities of risk prediction, ${ }^{22}$ and ensuring accurate understanding that patient confidentiality may be validly breached in situations of disclosures of self-harm or suicide

- effective approaches to reducing depression and suicidal behaviours in older primary care patients, including a program involving practice audit and personalised feedback, written materials, and educational newsletters, ${ }^{19}$ and providing GPs with clinical algorithms for treatment of depression in the elderly combined with depression care managers. ${ }^{23} \mathrm{~A}$ depression care manager supervised by a primary care expert and psychiatrist who provides education, support and practical guidance to GPs with the treatment of depression in late life (brief psychotherapy or medication) is also effective in reducing depressive symptoms, improving rates of depression treatment, and improving patient quality of life and function. ${ }^{24}$

\section{Support and sharing responsibility in care plans}

- While GPs are the coordinators of care for their older patients, this should not mean isolation and sole responsibility for managing their complex needs. Many of the multifactorial contributing factors to self-harm can be broken down into specific simple interventions to meet the patient's unique needs.

- Practice nurses and primary health networks (PHNs) may also have a key role in care coordination and follow-up.

- GP contact with families and carers should be encouraged, as they are underused informants and allies in treatment ${ }^{4,13}$ who could potentially co-manage the reversible factors for self-harm in older people and help monitor suicide risk. ${ }^{25}$

- GPs' sense of alienation and helplessness provide strong justification of the need for enhanced communication, including between specialist mental health services and primary care.

- Peer review groups and supervision (either with peers or psychiatrists) may provide a forum to present professional challenges and help prevent the development of negative attitudes and concern about failure of treatment. ${ }^{26}$

\section{Roles for primary health networks}

- The aim of the Australian Government's PHNs was to increase effectiveness and efficiency of medical services for patients through better coordination of care. ${ }^{27}$ Suicide prevention initiatives were specifically highlighted as targets for PHN focus in funding and practice. ${ }^{28}$ Primary mental healthcare activities targeted for funding included care coordination and clinical support, support for GPs with assessments and directing referrals to the most appropriate service for the patient's needs, and liaison with Local Health Networks and other organisations for follow-up care after a suicide attempt. ${ }^{29}$

- The PHN documents assert that GPs have a fundamental role in providing primary mental healthcare; in assessment, treatment, developing care plans and referral to other services commissioned by PHNs, ${ }^{29}$ which conflicts with GP responses here.

- As not all patients who self-harm want, can, or will receive mental health case management, one potential solution would be for the PHNs to develop care coordinator roles. The GP, having identified the contributory factors to their patient's self-harm, could then inform the care coordinator of individual patient needs to facilitate domiciliary assessments and support, specialist aged care, dementia and other clinical services, and appropriate follow-up and monitoring.

\section{Conclusion}

GPs have a broad understanding of why their patients may have self-harmed, and often have the benefit of a therapeutic relationship over many years. However, GPs did not nominate themselves as having a role in addressing the contributing factors to self-harm. Particular challenges identified were diagnosing and treating depression, especially with comorbid cognitive impairment, therapeutic nihilism and hopelessness. Educational interventions should target identified gaps in knowledge, practical pathways to address the complex biopsychosocial needs of older patients, and opportunities for better communication, support and referral to specialist services. Additionally, it is within the remit of PHNs to assist with care planning and coordination across services. The importance of engaging family carers in management is emphasised. We plan to test some of these strategies in future work on GP education about self-harm in older people.

\section{Authors}

Anne PF Wand FRANZCP, Staff Specialist Psychiatrist, Academic Department of Aged Care Psychiatry, Prince of Wales Hospital, Sydney, NSW; Conjoint Senior Lecturer, Discipline of Psychiatry, School of Medicine, University of New South Wales, Sydney, NSW. a.wand@unsw.edu.au

Carmelle Peisah FRANZCP, Conjoint Professor, Discipline of Psychiatry, School of Medicine, University of New South Wales, Sydney; Clinical Professor, Discipline of Psychiatry, Sydney Medical School, University of Sydney, Sydney, NSW; President, Capacity Australia, Crows Nest, NSW Brian Draper FRANZCP, Senior Staff Specialist Psychiatrist, Academic Department of Aged Care Psychiatry, Prince of Wales Hospital, Sydney, NSW; Conjoint Professor, Discipline of Psychiatry, School of Medicine, University of New South Wales, Sydney, NSW

Henry Brodaty FRANZCP, RACP, Scientia Professor, Dementia Collaborative Research Centre and Centre for Healthy Brain Ageing, University of New South Wales, Sydney, NSW

Competing interests: None.

Provenance and peer review: Not commissioned, externally peer reviewed.

\section{References}

1. De Leo D, Draper BM, Snowdon J, Kõlves K. Contacts with health professionals before suicide: Missed opportunities for prevention? Compr Psychiatry 2013;54(7):1117-23.

2. Shah A, Bhat R, Zarate-Escudero S, DeLeo D, Erlangsen A. Suicide rates in five-year age-bands after the age of 60 years: The international landscape. Aging Ment Health 2016;20(2):131-38.

3. Draper B, Brodaty H, Low LF. Types of nursing home residents with self-destructive behaviours: Analysis of the Harmful Behaviours Scale. Int J Geriatr Psychiatry 2002;17(7):670-75.

4. Draper B, Krysinska K, Snowdon J, De Leo D. Awareness of suicide risk and communication between health care professionals and next-of-kin of suicides in the month before suicide. Suicide Life Threat Behav 2017 [Epub ahead of print].

5. Uncapher H, Areán PA. Physicians are less willing to treat suicidal ideation in older patients. J Am Geriatr Soc 2000;48(2):188-92. 
6. Burroughs $H$, Lovell $K$, Morley M, Baldwin R, Burns A, Chew-Graham C. 'Justifiable depression': How primary care professionals and patients view late-life depression? A qualitative study. Fam Pract 2006;23(3):369-77.

7. Mullan M, Katona P, D'Ath P, Katona C. Screening, detection and management of depression in elderly primary care attenders. II: Detection and fitness for treatment: A case record study. Fam Pract 1994;11(3):267-70.

8. Livingston G, Yard P, Beard A, Katona C. A nursecoordinated educational initiative addressing primary care professionals' attitudes to and problem-solving in depression in older people - A pilot study. Int J Geriatr Psychiatry 2000;15(5):40105.

9. Braun V, Clarke V. Using thematic analysis in psychology. Qualitative Research in Psychology 2006;3:77-101.

10. Montano CB. Primary care issues related to the treatment of depression in elderly patients. J Clin Psychiatry 1999;60 Suppl 20:45-51.

11. Vannoy SD, Tai-Seale M, Duberstein P, Eaton LJ, Cook MA. Now what should I do? Primary care physicians' responses to older adults expressing thoughts of suicide. J Gen Intern Med 2011;26(9):1005-11.

12. Isometsä ET, Heikkinen ME, Marttunen MJ, Henriksson MM, Aro HM, Lönnqvist JK. The last appointment before suicide: Is suicide intent communicated? Am J Psychiatry 1995;152(6):919-22.

13. Kjølseth I, Ekeberg $\varnothing$. When elderly people give warning of suicide. Int Psychogeriatr 2012;24(9):1393-401.

14. Talseth AG, Gilje F, Norberg A. Being met - A passageway to hope for relatives of patients at risk of committing suicide: A phenomenological hermeneutic study. Arch Psychiatr Nurs 2001;15(6):249-56.
15. Freudenstein U, Jagger C, Arthur A, DonnerBanzhoff N. Treatments for late life depression in primary care - A systematic review. Fam Pract 2001;18(3):321-27.

16. Department of Health. Better access to psychiatrists, psychologists and general practitioners through MBS (Better Access initiative). Canberra: Commonwealth of Australia, 2017. Available at www.health.gov.au/ mentalhealth-betteraccess [Accessed 7 February 2018].

17. Dementia Support Australia. Dementia Support Australia (DBMAS \& SBRT) NSW. Sydney: DSA, date unknown. Available at www.agedcareguide. com.au/dementia-support-australia-dbmas-sbrtnsw [Accessed 28 October 2017].

18. Palmer V, Gunn J, Kokanovic R, et al. Diverse voices, simple desires: A conceptual design for primary care to respond to depression and related disorders. Fam Pract 2010;27(4):447-58.

19. Almeida OP, Pirkis J, Kerse N, et al. A randomized trial to reduce the prevalence of depression and self-harm behavior in older primary care patients. Ann Fam Med 2012;10(4):347-56.

20. Poma SZ, Grossi A, Toniolo E, Baldo V, Leo DD. Self-perceived difficulties with suicidal patients in a sample of Italian general practitioners. J Clin Med Res 2011;3(6):303-08.

21. Ritter K, Stompe T, Voracek M, Etzersdorfer E. Suicide risk-related knowledge and attitudes of general practitioners. Wien Klin Wochensch 2002;114(15-16):685-90.

22. Mulder R, Newton-Howes G, Coid JW. The futility of risk prediction in psychiatry. Br J Psychiatry 2016;209(4):271-72.

23. Bruce ML, Ten Have TR, Reynolds CF 3rd, et al. Reducing suicidal ideation and depressive symptoms in depressed older primary care patients: A randomized controlled trial. JAMA 2004;291(9):1081-91.
24. Unützer J, Katon W, Callahan CM, et al. Collaborative care management of latelife depression in the primary care setting: A randomized controlled trial. JAMA 2002;288(22):2836-45

25. Erlangsen A, Nordentoft M, Conwell Y, et al. Key considerations for preventing suicide in older adults: Consensus opinions of an expert panel. Crisis 2011;32(2):106-09.

26. Colson DB, Allen JG, Coyne L, et al. An anatomy of countertransference: Staff reactions to difficult psychiatric hospital patients. Hosp Community Psychiatry 1986;37(9):923-28.

27. Department of Health. PHN background. Canberra: DoH, 2015. Available at www.health.gov. au/internet/main/publishing.nsf/Content/PHNBackground [Accessed 4 August 2017].

28. Department of Health. Mental Health PHN Circular 1 - 12 January 2016. Canberra: DoH, 2016. Available at www.health.gov.au/internet/main/ publishing.nsf/Content/PHN-Circular1 [Accessed 4 August 17].

29. Department of Health. Annexure A1 Primary Mental Health Care. Canberra: DoH, updated 2017. Available at www.health.gov. au/internet/main/publishing.nsf/content/ F4F85B97E22A94CACA257F86007C7D1F [Accessed 6 August 2017].

correspondence ajgp@racgp.org.au 